SAND92-1460 U UC-405

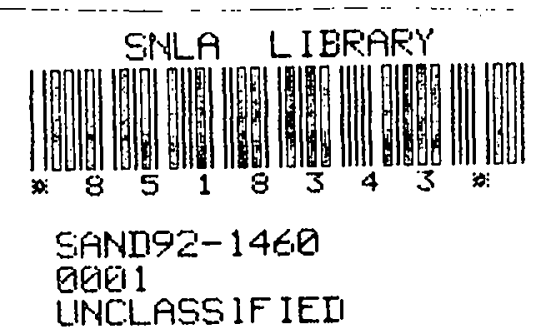

\title{
An Improved Spectral Graph Partitioning ¿EP Algorithm for Mapping Parallel Computations
} 019.92 STAC.

Bruce Hendrickson, Robert Leland

\section{Prepared by}

Sandia National Laboratories

Albuquerque, New Mexico 87185 and Livermore, California 94550

for the United States Department of Energy

under Contract DE-AC04-76DP00789

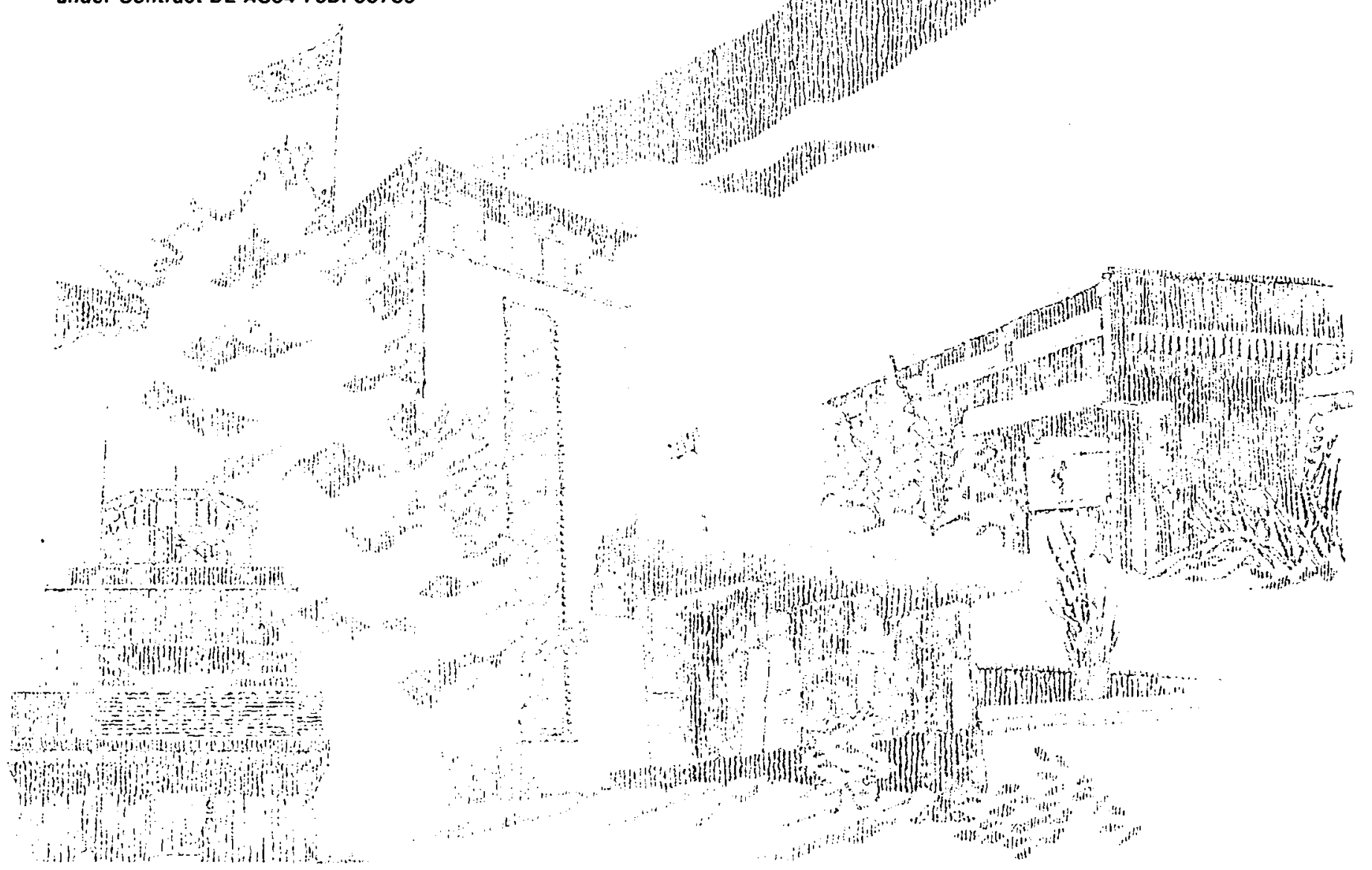


Issued by Sandia National Laboratories, operated for the United States Department of Energy by Sandia Corporation.

NOTICE: This report was prepared as an account of work sponsored by an agency of the United States Government. Neither the United States Government nor any agency thereof, nor any of their employees, nor any of their contractors, subcontractors, or their employees, makes any warranty, express or implied, or assumes any legal liability or responsibility for the accuracy, completeness, or usefulness of any information, apparatus, product, or process disclosed, or represents that its use would not infringe privately owned rights. Reference herein to any specific commercial product, process, or service by trade name, trademark, manufacturer, or otherwise, does not necessarily constitute or imply its endorsement, recommendation, or favoring by the United States Government, any agency thereof or any of their contractors or subcontractors. The views and opinions expressed herein do not necessarily state or reflect those of the United States Government, any agency thereof or any of their contractors.

Printed in the United States of America. This report has been reproduced directly from the best available copy.

Available to DOE and DOE contractors from

Office of Scientific and Technical Information

PO Box 62

Oak Ridge, TN 37831

Prices available from (615) 576-8401, FTS 626-8401

Available to the public from

National Technical Information Service

US Department of Commerce

5285 Port Royal Rd

Springfield, VA 22161

NTIS price codes

Printed copy: A03

Microfiche copy: A01 
SAND92-1460

Distribution

Unlimited Release

Category UC-405

Printed Setember 1992

\title{
An Improved Spectral Graph Partitioning Algorithm for Mapping Parallel Computations
}

\author{
Bruce Hendrickson and Robert Leland \\ Sandia National Laboratories \\ Albuquerque, NM 87185
}

\begin{abstract}
Efficient use of a distributed memory parallel computer requires that the computational load be balanced across processors in a way that minimizes interprocessor communication. We present a new domain mapping algorithm that extends recent work in which ideas from spectral graph theory have been applied to this problem. Our generalization of spectral graph bisection involves a novel use of multiple eigenvectors to allow for division of a computation into four or eight parts at each stage of a recursive decomposition. The resulting method is suitable for scientific computations like irregular finite elements or differences performed on hypercube or mesh architecture machines. Experimental results confirm that the new method provides better decompositions arrived at more economically and robustly than with previous spectral methods. We have also improved upon the known spectral lower bound for graph bisection.
\end{abstract}

This work was supported by the Applied Mathematical Sciences program, U.S. Department of Energy, Office of Energy Research, and was performed at Sandia National Laboratories, operated for the U.S. Department of Energy under contract No. DE-AC04-76DP00789. 
1. Introduction. Efficient use of a distributed memory parallel computer requires that the computational load be balanced across processors in a way that minimizes interprocessor communication. This mapping requirement can be abstracted to a graph problem in which nodes represent computation, edges represent communication, and the objective is to assign an equal number of vertices to each processor in a way that minimizes the number of edges crossing between processors. Extensive practical experience has shown that the quality of this mapping has a substantial impact on performance, hence there is considerable interest in effective mapping algorithms.

Finding a mapping that actually minimizes communication between balanced sets is an NP-hard problem [8], so it is unlikely that an efficient, general algorithm exists. The practical importance of this problem has, however, motivated a variety of heuristic approaches. These range from very quick, linear time algorithms based on geometric assumptions or local graph information to very slow algorithms which approximate a global search for the minimum using genetic operators or simulated annealing. The faster heuristics generally do not provide mappings of adequate quality for benchmarking purposes or for performance-critical codes which will be used many times, and the more expensive mapping techniques are impractical for use on large problems. This paper describes a method designed to provide near optimal partitionings at moderate cost.

Most existing load balancing methods are based on recursive graph bisection - the graph is broken in half, the halves are halved independently, and so on, until there are as many pieces as processors in the parallel machine. Bisection techniques have several inherent shortcomings. First, bisection algorithms are unable to accept a less attractive initial cut which would allow net savings in later cuts, i.e. they have no look ahead capability. In the VLSI community, for example, it is well known that recursive quadrisection leads to better circuit layouts than twice as many steps of bisection [19]. Second, in bisection the task of splitting the graph into sets of vertices (the decomposition problem) is largely decoupled from that of assigning a set of vertices to a specific processor (the assignment problem). The communication overhead of an application program, however, depends on both the decomposition and the assignment, so it is generally preferable to consider these aspects of the problem together. We might for example choose to accept a higher volume of communication between two sets in order to place them topologically closer on a given architecture.

Our approach to the graph partitioning problem addresses these shortcomings. It is based upon results from spectral graph theory, in which eigenvectors of a matrix are used to bisect a graph. The idea of using eigenvectors to partition graphs dates back to work in the early 70's by Donath and Hoffman [3, 4], and Fiedler [5, 6], but it has recently generated renewed interest $[2,13,15,16,17]$. Simon and Williams have applied spectral bisection to the load balancing problem and found it to have a number of attractive features in this context $[18,21]$. Unlike some other techniques, spectral methods are invariant under geometric transformations of the computational domain, as well as under renumbering of the computational graph. They also seem to generate good partitions in practice, albeit at a fairly high cost compared with some weaker 
heuristics.

Our method generalizes spectral graph bisection in several important ways. First, through a novel use of multiple eigenvectors, we are able to divide a problem into four or eight pieces at once instead of just two. This allows us to perform fewer recursive steps while dividing a problem into a given number of pieces. By trading off the combined effects of several cuts, we can reduce the look ahead problem associated with bisection. In addition, by using multiple eigenvectors we achieve a substantial economy in the net cost of the eigenvector calculations, the dominant expense. (Rendl and Wolkowicz describe a quite different spectral graph partitioning algorithm which requires $k$ eigenvectors to divide into $k$ sets rather than the $\log _{2} k$ eigenvectors we use [17].) Second, our model allows for inhomogeneous computation and communication requirements of an application, substantially broadening the class of problems for which it is appropriate. Third, our method does not ignore machine architecture, but rather explicitly accounts for hypercube topology in the communication cost. Recent empirical evidence confirms that this should lead to significantly better partitions in practice [9]. Our method can also be applied to meshes since $d$-dimensional meshes can be recursively decomposed as $d$-dimensional hypercubes. Fourth, unlike other approaches, our method solves the assignment problem simultaneously with the decomposition.

Our approach is designed for message passing multiprocessors, and is most appropriate for applications in which the computational requirements are static so that a good decomposition can be determined a-priori. It is particularly well suited to problems in which many different messages are simultaneously competing for use of the communication network. Many problems in scientific communication fit this description because they involve alternate phases of computation and communication in which the same calculation is repeated in each computation phase and many messages are transmitted in the communication phase. Examples include typical unstructured finite difference and finite element calculations.

The structure of this paper is as follows. In $\S 2$, we review hypercube multiprocessors, describe our graph model of computation and develop an associated metric of communication cost. This allows us to construct a discrete optimization problem which describes the optimal mapping in §3. Since this optimization problem is NP-hard, we derive a continuous problem approximating it in $\S 4$. In $\S 5$ and $\S 6$ we describe how the solution to this continuous problem reduces to an eigenvector calculation, and how the eigenvectors can be used to generate an approximate solution to the discrete optimization problem. Some new a-posteriori lower bounds on partition quality are presented in $\S 7$. Results of some sample calculations are given in $\S 8$, and conclusions are presented in $\S 9$.

\section{Preliminaries.}

2.1. Hypercube multiprocessors. A $d$-dimensional hypercube multiprocessor consists of a set of $2^{d}$ processors, identified by distinct binary numbers from 0 to $2^{d}-1$. Information is transmitted between them by passing messages through a network in which wires connect processors whose binary values differ in a single bit. We will 
assume there is no global memory, and that wires can simultaneously transmit data in either direction.

Hypercube multiprocessors enjoy wide popularity because they have attractive theoretical and practical properties. The network is very regular and can be described elegantly in a recursive fashion. Each processor is connected to just $d$ communication wires, and a message can be routed between any two processors by traversing at most $d$ wires. Furthermore, a message route can be devised simply; to travel between two processors, a message merely uses one wire from each bit in which the two processor's numbers differ.

2.2. A graph model of computation. As mentioned in $\S 1$, our approach to the partitioning problem is targeted mainly towards scientific computing applications. Most of these problems involve repeated iterations of the same cycle of computations. Although it is sometimes possible to achieve parallelism by effectively overlapping multiple iterations [22], the more common approach is to exploit parallelism within each iteration. Within an iteration, a processor performs a set of computations followed by a set of communication operations, and since each iteration involves the same set of operations, it is sufficient to distribute the task among processors based upon the requirements of a single iteration.

We represent a computation as an undirected, weighted graph $G=(V, E)$, using $n$ to denote the size of the vertex set $V$, and $m$ the size of the edge set $E$. Each vertex $v_{i} \in V$ corresponds to a computational task to be performed on a single processor, and the time required to execute that task is represented by a positive weight $w_{v}\left(v_{i}\right)$. We denote by $W_{v}$ the sum of the weights of all the vertices in the graph. An undirected edge $e_{i j} \in E$ connects two vertices $v_{i}$ and $v_{j}$ if the computational task represented by one of the vertices requires input from the other. The edge has an associated positive weight $w_{e}\left(e_{i j}\right)$ proportional to the amount of data that must be transmitted between the tasks. If each task requires data from the other, then this weight is the sum of the two amounts of information. The sum of the weights of all the edges is denoted by $W_{e}$. We will assume that $G$ is connected.

Partitioning a computational task among the processors corresponds to assigning each vertex of the graph to a processor. The sum of the weights of the vertices assigned to a processor represents the amount of computational effort that processor must expend, and the sum of the weights of all the edges connecting vertices assigned to two different processors represents the total amount of information that must be communicated between the two.

2.3. A communication metric. Most modern parallel computers have some form of hardware cut-through routing, so congestion aside, the time required to transmit a single message is nearly independent of the number of wires traversed. For applications like those we are targeting in which most messages are lengthy, this implies a model in which the communication cost of a message is proportional to the message length, independent of the identity of the sending and receiving processors. The cost of a set of messages can then be modeled as the sum of their individual costs. Within 
the constructs of our graph model, we define the cut-weight of a partitioning scheme to be the sum of the weights of all the edges whose vertices are assigned to different processors. Most previous approaches to domain mapping for parallel computing have tried to minimize this cut-weight communication measure.

However, since it treats messages in isolation, the cut-weight metric fails to consider any effects of message congestion. The applications we are considering typically have a communication phase in which there are many messages simultaneously competing for wires. In this case, each wire a message uses is unavailable for other tasks, so the load a message places on the network is proportional to the number of wires it consumes. Consequently we define the hop-weight of a message to be the length of the message multiplied by the number of wires it requires, and the hop-weight of a collection of messages to be the sum of their individual hop-weights. We will use hop-weight as our measure of the communication cost of a mapping. Recent experimental work has indicated that this is the most accurate communication metric for the problems we are targeting [9].

With the intent of making this discussion more formal, we let $\mathcal{M}: V \rightarrow P$ be an assignment scheme that maps vertices to processors. We denote by $\mathcal{V}(q)$ the set of vertices assigned to a processor $q$, so $\mathcal{V}(q)=\{v \in V: \mathcal{M}(v)=q\}$. We use $\rho_{i}$ to indicate the processor to which vertex $v_{i}$ is assigned, and $h_{i j}$ to denote the number of wires between $\rho_{i}$ and $\rho_{j}$. With this notation, we can formally define the hop-weight of an assignment as

$$
h o p-w e i g h t(\mathcal{M})=\sum_{e_{i j} \in E} w_{e}\left(e_{i j}\right) h_{i j}
$$

Next we map the binary digits designating a processor to \pm 1 by

$$
c^{k}(q)=\left\{\begin{aligned}
1 & \text { if the } k t h \text { bit of } q=1 \\
-1 & \text { if the } k t h \text { bit of } q=0
\end{aligned}\right.
$$

This transformation is convenient because the simple function $\left(1-c^{k}(q) c^{k}(r)\right) / 2$ is zero if processors $q$ and $r$ have the same $k$ th bit and one if they differ, and therefore is equal to $h_{r q}$. Hence the total communication cost on a hypercube under an assignment scheme $\mathcal{M}$ can be expressed as

$$
\operatorname{Cost}(\mathcal{M})=\text { hop-weight }(\mathcal{M})=\frac{1}{2} \sum_{e_{i j} \in E} \sum_{k=1}^{d} w_{e}\left(e_{i j}\right)\left(1-c^{k}\left(\rho_{i}\right) c^{k}\left(\rho_{j}\right)\right)
$$

We would like to find an assignment that minimizes this communication cost, while keeping the computational load balanced. We note that when $d=1$, hop-weight reduces to cut-weight, so in this case, the minimal cost is the bisection width of the graph.

3. A discrete optimization problem. When using a spectral method to solve a combinatorial problem, the general strategy is to formulate the combinatorial problem as a discrete optimization and then relax the discreteness constraint to obtain a continuous optimization problem. This continuous version may have some special structure 
making it tractable, even if the original discrete problem is NP-hard. After the continuous problem is solved, the result is mapped back to a nearby discrete point, which we hope provides a good approximation to the discrete optimum. A survey of results obtained using this general approach is given by Mohar in [13].

To follow this strategy we need to express our problem as a discrete optimization. The communication cost we wish to minimize is given in (3), but it will prove useful to add an additional term and interchange the order of summation to obtain

$$
\operatorname{Cost}(\mathcal{M})=\frac{1}{2} \sum_{k=1}^{d}\left\{\sum_{e_{i j} \in E} w_{e}\left(e_{i j}\right)\left(1-c^{k}\left(\rho_{i}\right) c^{k}\left(\rho_{j}\right)\right)+\frac{1}{2} \sum_{i=1}^{n} t_{i}\left(c^{k}\left(\rho_{i}\right)^{2}-1\right)\right\} .
$$

Since $c^{k}(q)= \pm 1$, this last term is zero, and does not change the value of $\operatorname{Cost}(\mathcal{M})$. However, when the discreteness constraint on $c^{k}$ is relaxed in $\S 4$, this term will become important. Appropriate values for $t_{i}$ will also be considered in $\S 4$.

Equation (4) describes the communication cost to be minimized, but it must be constrained to ensure load balance. The computational load is balanced if the sums of the weights of the vertices assigned to each processor are equal. Denote by $\mathcal{W}(q)$ the sums of weights of all vertices assigned to processor $q$, so that $\mathcal{W}(q)=\sum_{v \in \mathcal{V}(q)} w_{v}(v)$. The load balance constraint can then be expressed as

$$
\mathcal{W}(q)=W_{v} / 2^{d}, \quad \forall q \in\left\{0, \ldots, 2^{d}-1\right\} .
$$

It will prove convenient to use a different form of the balance constraint expressed in terms of the $c^{k}$ notation introduced in (2). In particular, the conditions

$$
\begin{aligned}
& \sum_{k=0}^{2^{d}-1} \mathcal{W}(k)=W_{v} \\
& \sum_{k=0}^{2^{d-1}} \mathcal{W}(k) \prod_{j \in \mathcal{S}} c^{j}(k)=0, \quad \forall \mathcal{S}: \emptyset \neq \mathcal{S} \subseteq\{1, \ldots, d\} .
\end{aligned}
$$

ensure balance, as demonstrated by Theorem 3.1 .

ThEOREM 3.1. Equations (6) and (5) are equivalent.

Proof. Given a set $S$ of size $d$, we can represent any subset of $S$ as a binary string in which 1 indicates the presence and 0 indicates the absence of the corresponding set element. Hence there are $2^{d}$ subsets corresponding to the possible bit patterns. Condition (6b) excludes the null subset and so provides $2^{d}-1$ constraint equations. With the addition of $(6 \mathrm{a})$ we therefore have $2^{d}$ linearly independent equations for the $2^{d}$ unknown values of $\mathcal{W}(k)$, so a unique solution exists. Since the solution to (5) is easily seen to be a solution to (6), the two formulations of constraint equations are equivalent.

A still more convenient formulation of the balance constraint involves the $w_{v}\left(v_{i}\right)$ values and has the form

$$
\sum_{i=1}^{n} w_{v}\left(v_{i}\right) \prod_{j \in \mathcal{S}} c^{j}\left(\rho_{i}\right)=0, \quad \forall \mathcal{S}: \emptyset \neq \mathcal{S} \subseteq\{1, \ldots, d\} .
$$


Equation (6a) is automatically satisfied by the $w_{v}\left(v_{i}\right)$ values, and the equivalence of (7) and $(6)$ is a straightforward consequence of the definitions.

Combining (4) with (7) we obtain a formal statement of the problem of minimizing communication subject to the load balance constraint.

Minimize $\frac{1}{2} \sum_{k=1}^{d}\left\{\sum_{e_{i} \in E} w_{e}\left(e_{i j}\right)\left(1-c^{k}\left(\rho_{i}\right) c^{k}\left(\rho_{j}\right)\right)+\frac{1}{2} \sum_{i=1}^{n} t_{i}\left(c^{k}\left(\rho_{i}\right)^{2}-1\right)\right\}$

Subject to :

(a) $\quad c^{k}(q)= \pm 1, \quad \forall k \in\{1, \ldots, d\}, \forall q \in\left\{0, \ldots, 2^{d}-1\right\}$

$$
\sum_{i=1}^{n} w_{v}\left(v_{i}\right) \prod_{j \in \mathcal{S}} c^{j}\left(\rho_{i}\right)=0, \quad \forall \mathcal{S}: \emptyset \neq \mathcal{S} \subseteq\{1, \ldots, d\}
$$

We will call this discrete optimization problem P1. It is NP-hard since it generalizes the problem of graph bisection [8]. A general, efficient algorithm for solving it is therefore unlikely to exist, and we are forced to resort to heuristics.

4. A continuous approximation. Since solving (P1) is difficult, we approximate it by an easier problem. In particular, we relax the constraint that $c^{k}(q)= \pm 1$, which changes the discrete problem into a tractable continuous optimization problem. Unfortunately the solution to the continuous problem does not give us a valid partitioning since the $c^{k}(q)$ 's will no longer have discrete values corresponding to the bit patterns of the target processors. We can, however, use the solution of the continuous optimization to find a nearby point satisfying the \pm 1 condition. This nearby point will not generally be the absolute minimizer of $(\mathbf{P 1})$, but the hope is that it will provide a good answer in practice.

It will be convenient to reformulate (P1) in matrix terms. For a fixed $k$, consider the $n$ values of $c^{k}\left(\rho_{i}\right) \forall i \in\{1, \ldots, n\}$ as an $n$-vector denoted by $x^{k}$. Introduce the weighted adjacency matrix $A$ as follows.

$$
A(i, j)=\left\{\begin{array}{cl}
w_{e}\left(e_{i j}\right) & \text { if } e_{i j} \in E \\
0 & \text { Otherwise. }
\end{array}\right.
$$

Letting $D=\operatorname{Diag}\left(t_{i}\right)$ and $\tau=\sum_{i=1}^{n} t_{i}$, the objective function in $(\mathbf{P} 1)$ can be rewritten in matrix notation as

$$
\frac{1}{2} d\left(W_{e}-\frac{\tau}{2}\right)+\frac{1}{4} \sum_{k=1}^{d}\left(x^{k}\right)^{T} B x^{k}
$$

where $\left(x^{k}\right)^{T}$ denotes the transpose of $x^{k}$, and $B=D-A$. We note that the leading constant term has no effect on the minimizer, just on the minimum value.

We set the diagonal values $t_{i}$ to make each row sum of $B$ zero. There is no compelling reason for this choice, but it is convenient for several reasons. First, since $t_{i}=\sum_{e_{i j} \in E} w_{e}\left(e_{i j}\right)$ implies that $\tau=2 W_{e}$, the initial term in the cost is identically zero. Second, the matrix $B$ is positive semidefinite, and if the graph is connected then $B$ has only a single null vector consisting of all 1's. Third, if the edge weights are all 1 , then 
$B$ reduces to the familiar Laplacian matrix of the graph - our matrix is a weighted Laplacian. We expect this to be advantageous because unweighted Laplacians have proved useful in a number of combinatorial optimization problems [13]. In particular, when used to partition graphs into two sets (a special case of what we will describe below), the Laplacian facilitates several theoretical results $[2,5,6]$. Fourth, this choice is convenient for solving the eigenvector problem arising below.

Now we relax the constraint that each of the elements of the $x^{k}$ vectors must be \pm 1 . Instead, we impose the norm constraint $\left\|x^{k}\right\|_{2}=\sqrt{n}$. Combining this continuous constraint with (8), (10) and the expression for $\tau$ yields the following continuous approximation to $(\mathbf{P 1})$.

$$
\text { Minimize } \frac{1}{4} \sum_{k=1}^{d}\left(x^{k}\right)^{T} B x^{k}
$$

Subject to :

$$
\begin{aligned}
& x^{k} \in \mathbb{R}^{n}, \quad \forall k \in\{1, \ldots, d\} \\
& \left(x^{k}\right)^{T} x^{k}=n, \quad \forall k \in\{1, \ldots, d\} \\
& \sum_{i=1}^{n} w_{v}\left(v_{i}\right) \prod_{j \in \mathcal{S}} x^{j}(i)=0, \quad \forall \mathcal{S}: \emptyset \neq \mathcal{S} \subseteq\{1, \ldots, d\} .
\end{aligned}
$$

We call this problem (P2) and note that its solution provides a lower bound for the solution of $(\mathbf{P 1} 1)$. The advantage of approximating (P1) by (P2) is that the latter can be solved efficiently, as the next section will demonstrate.

5. Solving the continuous approximation. To solve (P2) we begin by focusing on a subset of the constraints. Instead of considering all of the terms of $(11 \mathrm{~b})$, we will concentrate on only those terms involving 2 or fewer elements in the products. These terms are

$$
\begin{aligned}
& \sum_{i=1}^{n} w_{v}\left(v_{i}\right) x^{k}(i)=0, \quad \forall k \in\{1, \ldots, d\} \\
& \sum_{i=1}^{n} w_{v}\left(v_{i}\right) x^{k}(i) x^{j}(i)=0, \quad \forall k, j \in\{1, \ldots, d\}: k \neq j .
\end{aligned}
$$

We note that if $d=1$ (bisection), this second constraint is irrelevant, and if $d=2$ (quadrisection) these are the only constraints in (11b).

To simplify, we change variables to a set of vectors $y^{k} \in \mathbb{R}^{n}$ defined by $y^{k}(i)=$ $\sqrt{w_{v}\left(v_{i}\right)} x^{k}(i)$. Since the $x^{k}$ values are relaxations of \pm 1 , the appropriate normalization for the $y$ vectors is $\left(y^{k}\right)^{T} y^{k}=W_{v}$. Letting $s, \bar{s} \in \mathbb{R}^{n}$ be vectors in which $s_{i}=\sqrt{w_{v}\left(v_{i}\right)}$, and $\bar{s}_{i}=1 / \sqrt{w_{v}\left(v_{i}\right)},(12)$ is transformed to

$$
\begin{aligned}
& s^{T} y^{k}=0, \quad \forall k \in\{1, \ldots, d\} \\
& \left(y^{k}\right)^{T} y^{j}=0, \quad \forall k, j \in\{1, \ldots, d\}: k \neq j .
\end{aligned}
$$


Combining (13) with (11), and letting $C=\operatorname{Diag}(\bar{s})^{T} B \operatorname{Diag}(\bar{s})$, we can rewrite (P2) as

Minimize $\frac{1}{4} \sum_{k=1}^{d}\left(y^{k}\right)^{T} C y^{k}$

Subject to :

(a1) $\quad y^{k} \in \mathbb{R}^{n}, \quad \forall k \in\{1, \ldots, d\}$

(a2) $\quad\left(y^{k}\right)^{T} y^{k}=W_{v}, \quad \forall k \in\{1, \ldots, d\}$

(b1) $\quad s^{T} y^{k}=0, \quad \forall k \in\{1, \ldots, d\}$

(b2) $\quad\left(y^{k}\right)^{T} y^{j}=0, \quad \forall k, j \in\{1, \ldots, d\}: k \neq j$

$$
\sum_{i=1}^{n} w_{v}\left(v_{i}\right)^{1-|\mathcal{S}| / 2} \prod_{j \in \mathcal{S}} y^{j}(i)=0, \quad \forall \mathcal{S}: \mathcal{S} \subseteq\{1, \ldots, d\},|\mathcal{S}|>2,
$$

which we denote by (P3). Next we collect a number of useful observations regarding the matrix $C$.

THEOREM 5.1. The matrix $C$ has the following properties.

(I) $C$ is symmetric positive semidefinite.

(II) The eigenvectors of $C$ can always be chosen to be pairwise orthogonal.

(III) The vector $s$ is an eigenvector of $C$ with eigenvalue zero.

(IV) If the graph is connected, $s$ is the only eigenvector of $C$ with eigenvalue zero.

Proof. Orient each edge in the graph arbitrarily and define the standard incidence matrix of a graph $F \in \mathbb{R}^{n \times m}$ such that

$$
F(i, l)=\left\{\begin{aligned}
1 & \text { if } i \text { is the initial vertex of the lth edge, } \\
-1 & \text { if } i \text { is the terminal vertex of the lth edge } \\
0 & \text { if } i \text { is not incident to the lth edge. }
\end{aligned}\right.
$$

Now define a weighted incidence matrix $G \in \mathbb{R}^{n \times m}$ as $G=\operatorname{Diag}(\bar{s}) F \operatorname{Diag}\left(\sqrt{w_{e}\left(e_{l}\right)}\right)$. Property (I) follows from the observation that $C$ can be written as $G G^{T}$. Property (II) is a consequence of the symmetry of $C$ and acknowledges that $C$ may have multiple eigenvalues. The observation that the vector of all ones is a zero eigenvector of $B$ yields (III). Property (IV) follows from Theorem $2.1 \mathrm{c}$ of [13].

We now define one further minimization problem, denoted by $(\mathbf{P 4})$, to be the same as (P3) but with constraint (b3) removed. This is useful because (P4) can be solved easily, and its solution can then be used to solve (P3). (In fact, (P3) and (P4) are equivalent if $d \leq 2$ ). We define $u^{i}$ to be the normalized eigenvectors of $C$ with corresponding nondecreasing eigenvalues $\lambda_{i}$. The solution to (P4) is easily expressed in terms of these eigenvectors as the following lemmas and theorem demonstrate.

LEMMA 5.2. Let $y^{1}, \ldots, y^{d}$ be a set of vectors that solves $(\mathbf{P 4})$, and denote the span of the $y^{k}$ vectors by $\mathcal{Y}$. Then any set of orthogonal vectors $z^{k}$ that spans $\mathcal{Y}$ and satisfies $\left\|z^{k}\right\|_{2}=\sqrt{W_{v}}, \forall k \in\{1, \ldots, d\}$ also solves $(\mathbf{P 4})$.

Proof. The objective function in $(\mathbf{P} 4)$ can be written as $\operatorname{trace}\left(Y^{T} C Y\right)$, where $Y$ is the $n \times d$ matrix whose $k$ th column is the vector $y^{k}$. Any orthogonal basis $Z$ for 
$\mathcal{Y}$ that satisfies the normalization constraint can be written as $Z=Y R$, where $R$ is an orthonormal matrix. We now observe that $\operatorname{trace}\left(Z^{T} C Z\right)=\operatorname{trace}\left(R^{T} Y^{T} C Y R\right)=$ $\operatorname{trace}\left(Y^{T} C Y\right)$, and the lemma follows.

LEMMA 5.3. If $y^{1}, \ldots, y^{d}$ solves $(\mathbf{P 4})$, then there is another set of vectors $\bar{z}^{1}, \ldots, \bar{z}^{d}$ in which $\left(\bar{z}^{k}\right)^{T} u^{i}=0$, if $k \geq i$ that also solves (P4)

Proof. Theorem 5.1(III) coupled with constraint (b1) ensures that $\left(y^{k}\right) u^{1}=0$, for all $k$. Starting with the $y^{k}$ vectors, we can apply rotations to satisfy the remaining orthogonality constraints. Lemma 5.2 ensures that the value of the objective function is invariant with respect to these rotations.

THEOREM 5.4. Any set of orthogonal vectors $y^{1}, \ldots, y^{d}$ that spans $\left\{u^{2}, \ldots, u^{d+1}\right\}$ and satisfies $\left\|y^{k}\right\|_{2}=\sqrt{W_{v}}, \forall k \in\{1, \ldots, d\}$ solves (P4). Furthermone, if $\lambda_{d+1}<\lambda_{d+2}$, then these are the only solutions to (P4).

Proof. By Lemma 5.3, there is a solution $\bar{z}^{k}$ to $(\mathbf{P 4})$ in which $\left(\bar{z}^{k}\right)^{T} u^{i}=0$, if $k \geq i$. Since $C$ is symmetric, we can rewrite $\bar{z}^{k}$ as a linear sum of eigenvectors of $C: \bar{z}^{k}=$ $\sum_{i=1}^{n} \alpha_{i}^{k} u^{i}$. Now the objective function can be written as

$$
4 \text { Cost }=\sum_{k=1}^{d}\left(\bar{z}^{k}\right)^{T} C \bar{z}^{k}=\sum_{k=1}^{d} \sum_{i=1}^{n}\left(\alpha_{i}^{k}\right)^{2} \lambda_{i} .
$$

Constraint (a2) implies $\sum_{i=1}^{n}\left(\alpha_{i}^{k}\right)^{2}=W_{v}$ for all $k$, and the construction of the $\bar{z}^{k}$ vectors guarantees that $\alpha_{i}^{k}=0$, if $k \geq i$. Using these identities we can rewrite the cost function as follows.

$$
\begin{aligned}
4 \text { Cost } & =\sum_{k=1}^{d} \sum_{i=k+1}^{n}\left(\alpha_{i}^{k}\right)^{2} \lambda_{i} \\
& \geq \sum_{k=1}^{d} \lambda_{k+1} \sum_{i=k+1}^{n}\left(\alpha_{i}^{k}\right)^{2} \\
& =\sum_{k=1}^{d} \lambda_{k+1} W_{v}
\end{aligned}
$$

It is easy to verify that this lower bound can be achieved by letting $\bar{z}^{k}=\sqrt{W_{\nu}} u^{k+1}$. By applying Lemma 5.2, we conclude that all orthogonal bases $y^{1}, \ldots, y^{d}$ for the space spanned by $\left\{u^{2}, \ldots, u^{d+1}\right\}$ in which $\left\|y^{k}\right\|_{2}=\sqrt{W_{v}}$ solve (P4).

To see that these are the only solutions to (P4) it is sufficient to observe that if $\lambda_{d+1}<\lambda_{d+2}$, the inequality between (17) and (18) is strict unless $\alpha_{i}^{k}=0$, when $i>d+1$. But this implies the $\bar{z}^{k}$ vectors lie in the space spanned by $\left\{u^{2}, \ldots, u^{d+1}\right\}$, and the theorem follows.

Theorem 5.4 indicates that if $d>1$, there is a space of solutions to (P4) of dimension $\left(\begin{array}{l}d \\ 2\end{array}\right)$. This multiplicity of solutions is quite convenient since the continuous solution is only an approximation to the the discrete problem (P1). If the continuous optimization had only a single minimizer and that minimizer was far from any of the discrete points then the continuous problem could be a poor model of the discrete one. Since we have a $d$-dimensional subspace of minimizers, we have a better chance of finding a good discrete solution. These degrees of freedom also allow us to satisfy the additional constraints of (P3). 
5.1. Spectral bisection. If we wish to divide our graph into two pieces, then (P4) reduces to (P3) since constraints (b2) and (b3) have no effect. We therefore take $y^{1}$ to be $\sqrt{W_{v}} u^{2}$, and let $x^{1}(i)=y^{1}(i) / \sqrt{w_{v}\left(v_{i}\right)}$. The vector $x^{1}$ is the continuous approximation to \pm 1 values, so we need to map it to a nearby discrete point with an equal weight of +1 and -1 values. We do this by finding the median weighted value among all the $x^{1}(i)$ 's and mapping values above the median to +1 and values below to -1 . This gives a balanced decomposition with, hopefully, a low cut-weight.

Once the graph is divided into two pieces, each piece can be divided again by applying this technique recursively. For unweighted graphs, this is the partitioning procedure described by Pothen, Simon and Liou in [15] and first applied to the load balancing problem by Simon [18]. Simon found this approach to produce better partitions than several other popular methods.

5.2. Spectral quadrisection. Dividing the graph into four pieces requires two eigenvectors. With two eigenvectors the constraint (14b3) is unnecessary, so (P4) is again equivalent to (P3). The solutions of (P4) are any appropriately normalized orthogonal basis for the space spanned by $y^{1}=\sqrt{W_{v}} u^{2}$ and $y^{2}=\sqrt{W_{v}} u^{3}$. This multiplicity of solutions allows us a single rotational degree of freedom, which yields vectors of the form $\bar{y}^{1}=y^{1} \cos \theta+y^{2} \sin \theta$, and $\bar{y}^{2}=-y^{1} \sin \theta+y^{2} \cos \theta$. From the $\bar{y}$ vectors we generate $x$ vectors whose values approximate \pm 1 by $x^{k}(i)=\bar{y}^{k}(i) / \sqrt{w_{v}\left(v_{i}\right)}$. Ideally, we would like to find $\bar{y}$ vectors in which the corresponding $x$ values are near to points with values \pm 1 to help ensure that the cost of the discrete solution is not too different from the continuous optimum.

The distance from $x^{k}(i)$ to \pm 1 can be expressed as $\left(1-x^{k}(i)^{2}\right)^{2}$. Summing over each element of both $k$ vectors, we find that we must solve

$$
\text { Minimize } \sum_{i=1}^{n} \sum_{k=1}^{2}\left(1-x^{k}(i)^{2}\right)^{2}
$$

Expanding $x^{k}(i)$ in terms of $\theta,(19)$ reduces to minimizing a constant coefficient quartic equation in sines and cosines of $\theta$. The construction of the coefficients in this equation requires $O(n)$ work, but the cost of the resulting minimization problem is independent of $n$. Although this is a global optimization problem, in our experience the number of local minimizers is small, so a solution can be found by a sequence of local minimizations from random starting points [11].

Once $x^{1}$ and $x^{2}$ have been determined, a nearby discrete point must be found that balances the partition sizes. Our solution to this problem is described in $\S 6$.

5.3. Spectral octasection. Dividing the graph into eight pieces requires three eigenvectors. In this case, the constraints (14b1) and (14b2) are insufficient, since (14b3) generates an additional cubic constraint of the form

$$
\sum_{i=1}^{n} y^{1}(i) y^{2}(i) y^{3}(i) / \sqrt{w_{v}\left(v_{i}\right)}=0
$$


As before, the solutions of (P4) are any appropriately normalized orthogonal basis for the space spanned by $y^{1}=\sqrt{W_{v}} u^{2}, y^{2}=\sqrt{W_{v}} u^{3}$ and $y^{3}=\sqrt{W_{v}} u^{4}$, but these are not necessarily solutions of (P3). The additional constraint (14b3) removes one degree of freedom from the three-dimensional solution space for (P3), leaving a two-dimensional parameter space to explore.

As in $§ 5.2$, we use these remaining degrees of freedom to look for $\bar{y}$ vectors that generate $x$ values as near as possible to \pm 1 . The bases for the eigenspace $\bar{y}$ can be described in terms of three rotational parameters. The $\bar{y}^{k}$ vectors are mapped to $x^{k}$ vectors by $x^{k}(i)=\bar{y}^{k}(i) / \sqrt{w_{v}\left(v_{i}\right)}$. This generates a constrained optimization problem

$$
\begin{aligned}
& \text { Minimize } \sum_{i=1}^{n} \sum_{k=1}^{3}\left(1-x^{k}(i)^{2}\right)^{2} \\
& \text { Subject to : } \\
& \qquad \sum_{i=1}^{n} w_{v}\left(v_{i}\right) x^{1}(i) x^{2}(i) x^{3}(i)=0 .
\end{aligned}
$$

in which the objective function is a constant coefficient polynomial in sines and cosines of three angular parameters. The coefficients can be generated in $O(n)$ time, after which the cost of the optimization problem is independent of $n$. As before this is a global optimization problem, but in our experience the number of local minimizers is small, so a solution can be found by a sequence of constrained local minimizations from random starting points [7].

As in $\S 5.2$, once $x^{1}, x^{2}$ and $x^{3}$ have been determined, a nearby discrete point must be found that balances the partition sizes. Our method for solving this problem is described in $\S 6$.

5.4. Higher order partitionings. When $d>3$ the partitioning problem becomes more difficult. The subspace defined by the set of eigenvectors of $C$ will allow $\left(\begin{array}{l}d \\ 2\end{array}\right)$ degrees of rotational freedom. However, there will be a set of $\left(\begin{array}{l}d \\ 3\end{array}\right)+\cdots+\left(\begin{array}{l}d \\ d\end{array}\right)$ constraints due to $(14 \mathrm{~b} 3)$. When $d>4$, there are more constraints than degrees of freedorn, so it will not generally be possible to construct a balanced solution from the $d+1$ lowest eigenvectors of $C$. When $d=4$ there are six variables and five constraints, so it should be possible to satisfy all the balance conditions. However, these constraints consist of three cubic equations and one quartic, so the computational complexity of satisfying them is daunting. For this reason we have chosen not to implement any partitioning above octasection, and we suggest recursive application of one of the above schemes to divide a problem across a larger number of processors.

6. Generating a partition from real values. The procedures described in $\S 5.2$ and $\S 5.3$ generate a point in $\mathbb{R}^{d}$ for each vertex in the graph. These continuous points need to be mapped to points with coordinates \pm 1 to determine a partition. This mapping must ensure that equal weights of vertices are assigned to each partition, and each continuous value should be mapped to a nearby discrete point.

It is useful to describe this mapping problem in terms of a complete, weighted bipartite graph $\mathcal{B}=\left\{V_{1}, V_{2}, \mathcal{E}\right\}$. The first set of vertices $V_{1}$ consists of the $n$ vertices 
of our original graph, while the second set $V_{2}$ corresponds to the $2^{d}$ sets. A weighted edge $e \in \mathcal{E}$ connects each vertex $x \in V_{1}$ to each vertex $y \in V_{2}$, with weight equal to the distance between the continuous point corresponding to $x$ and the discrete point associated with the set $y$. Any distance function can be used, but we chose the square of the Euclidean distance for computational convenience. There is also a vertex weight associated with each vertex $x \in V_{1}$, equal to the weight of the corresponding vertex in the original graph.

The optimal mapping can now be described in terms of a minimum cost assignment from $V_{1}$ to $V_{2}$ with the constraint that the sums of the vertex weights of the elements of $V_{1}$ mapped to each element of $V_{2}$ are equal. This is a generalization of a class of assignment problems considered by Tokuyama and Nakano [20], who develop an assortment of algorithms that generalize in a straightforward manner to our problem. Their best algorithm is randomized and requires $O\left(2^{d} n\right)$ time. We chose instead to implement one of their simpler, deterministic algorithms that runs in $O\left(2^{2 d-1} n \log n\right)$ time. By exploiting the geometric structure of our particular application it is possible to reduce this time bound to $O\left(3^{d} n \log n\right)$.

7. Lower bounds on partitions. A known bound on the edge count for bisection of an unweighted graph is $\frac{1}{4} n \lambda_{2}$, where $\lambda_{2}$ is the second lowest eigenvalue of the Laplacian matrix of the graph (see for example [13]). A simple consequence of the results in $\S 5$ is a generalization of this bound with respect to both weighting and dimensionality.

THEOREM 7.1. The communication cost induced by cutting a graph into $2^{d}$ pieces is always at least $\frac{1}{4} W_{v} \sum_{i=2}^{d+1} \lambda_{i}$.

Proof. Since (P4) is derived from (P1) by relaxing constraints, the minimum of (P4) will never be larger than that of (P1). Substitution of the solution in Theorem 5.4 into the cost function of (14) leads to the result.

A better bisection bound can be determined by considering the difference between the continuous and discrete solution vectors. The continuous solution is the vector $y^{1}=\sqrt{W_{v}} u^{2}$ from $\S 5.1$. We let $b \in \mathbb{R}^{n}$ be the vector with the smallest 2 -norm among all vectors such that $y^{1}(i)+b(i)= \pm \sqrt{w_{v}\left(v_{i}\right)}$, and let $\beta=\|b\|_{2}^{2}$. We note that $b$ (and consequently $\beta$ ) is easy to compute using

$$
b(i)=\min \left\{y^{1}(i)-\sqrt{w_{v}\left(v_{i}\right)}, y^{1}(i)+\sqrt{w_{v}\left(v_{i}\right)}\right\}
$$

THEOREM 7.2. The bisection width of a graph is bounded by

$$
\text { Cost } \geq \frac{1}{4}\left\{W_{v} \lambda_{2}+\left(\lambda_{3}-\lambda_{2}\right) \beta\left(1-\frac{\beta}{4 W_{v}}\right)\right\} .
$$

Proof. If $c \in\{ \pm 1\}^{n}$ is the discrete solution to (P1), define $z$ to be its weighted counterpart, $z(i)=\sqrt{w_{v}\left(v_{i}\right)} c(i)$. We note that if $z$ defines a partition, then $-z$ defines the same partition, so without loss of generality we can assume that $z^{T} y^{1} \geq 0$. We define $a \in \mathbb{R}^{n}$ to be the difference between $z$ and $y^{1}$, so $a(i)=z(i)-y^{1}(i)$. We can expand $a$ in terms of the eigenvectors of $C$ so that $a=\sum_{j=2}^{n} \alpha_{j} u_{j}$, where this expansion begins at 2 
since $a$ is orthogonal to $u_{1}$. It follows from the definition of $\beta$ that $\beta \leq a^{T} a=\sum_{j=2}^{n} \alpha_{j}^{2}$. Now

$$
\begin{aligned}
W_{v}=z^{T} z & =\left(y^{1}+a\right)^{T}\left(y^{1}+a\right) \\
& =\left(y^{1}\right)^{T} y^{1}+2 a^{T} y^{1}+a^{T} a \\
& =W_{v}+2 \sqrt{W_{v}} \alpha_{2}+a^{T} a
\end{aligned}
$$

so $\alpha_{2}=-a^{T} a /\left(2 \sqrt{W_{v}}\right)$. Since $0 \leq z^{T} y^{1}=\left(y^{1}+a\right)^{T} y_{1}=W_{v}+\sqrt{W_{v}} \alpha_{2}$, it follows that $\alpha_{2} \geq-\sqrt{W_{v}}$, which implies that $a^{T} a \leq 2 W_{v}$.

The bisection width of the graph can be expressed as

$$
\begin{aligned}
4 \text { Cost } & =z^{T} C z \\
& =\left(y^{1}+a\right)^{T} C\left(y^{1}+a\right) \\
& =\left(y^{1}\right)^{T} C y^{1}+2 a^{T} C y^{1}+a^{T} C a \\
& =W_{v} \lambda_{2}+2 \sqrt{W_{v}} \lambda_{2} \alpha_{2}+\sum_{j=2}^{n} \lambda_{j} \alpha_{j}^{2} \\
& =W_{v} \lambda_{2}-\lambda_{2} a^{T} a+\sum_{j=2}^{n} \lambda_{j} \alpha_{j}^{2} \\
& =W_{v} \lambda_{2}+\sum_{j=2}^{n}\left(\lambda_{j}-\lambda_{2}\right) \alpha_{j}^{2}
\end{aligned}
$$

The sum in the second term of (26) is minimized when $\alpha_{j}=0$ for all $j>3$, in which case $\alpha_{3}^{2}=a^{T} a-\alpha_{2}^{2}=a^{T} a\left(1-a^{T} a /\left(4 W_{v}\right)\right)$. This implies that

$$
4 \text { Cost } \geq W_{v} \lambda_{2}+\left(\lambda_{3}-\lambda_{2}\right) a^{T} a\left(1-a^{T} a /\left(4 W_{v}\right)\right) \text {. }
$$

This last term comprises a concave function in $a^{r} a$, so its minimum value occurs when $a^{T} a$ is either maximized or minimized. Using the above observations that $\beta \leq a^{T} a \leq$ $2 W_{v}$, we obtain

$$
4 \text { Cost } \geq \min \left\{W_{v} \lambda_{2}+\left(\lambda_{3}-\lambda_{2}\right) W_{v}, W_{v} \lambda_{2}+\left(\lambda_{3}-\lambda_{2}\right) \beta\left(1-\beta /\left(4 W_{v}\right)\right)\right\}
$$

But since $\beta\left(1-\beta /\left(4 W_{v}\right)\right)$ has a maximum value of $W_{v}$, the second term of (28) always dominates and the theorem follows.

Although the bound in Theorem 7.2 is better than previously known spectral bounds, it is still rather loose in practice and its practical value is therefore not clear. It may help in identifying classes of graphs for which the spectral method achieves near optimal results, or for proving that some particular graphs have large bisection widths.

8. Results. We have compared the quality of partitions produced by our algorithm with those generated by several other graph partitioning methods which are in common use or have been recently advocated. Our conclusion is that the improved spectral partitioning algorithm we have proposed generates significantly better partitions than these other methods, which are themselves considered to be quite good. This 
is based on direct experimental comparison using a variety of meshes. We have selected one representative test for this paper, a finite element meshing of a multi-element airfoil provided by Barth [1]. A more comprehensive reporting of our experimental results is contained in the follow on paper [10].

The airfoil mesh is shown in Figure 1 and its dual is shown in Figure 2. The dual has a vertex representing each element in the mesh (triangular faces in this case) and an edge connecting vertices representing elements which share an edge in the mesh. There are 8034 vertices and 11813 edges in this dual graph. The dual is relevant because in many parallel finite element codes, data is organized by assigning collections of individual elements to each processor. The iterative solution of the resulting equations then involves some computation associated with each element and some communication between elements sharing an edge or vertex. The dual graph therefore provides a better model for the iterative solution than the original mesh does. For ease of comparison with other methods, we chose to partition an instance of the dual in which all vertex and edge weights are equal to 1 .

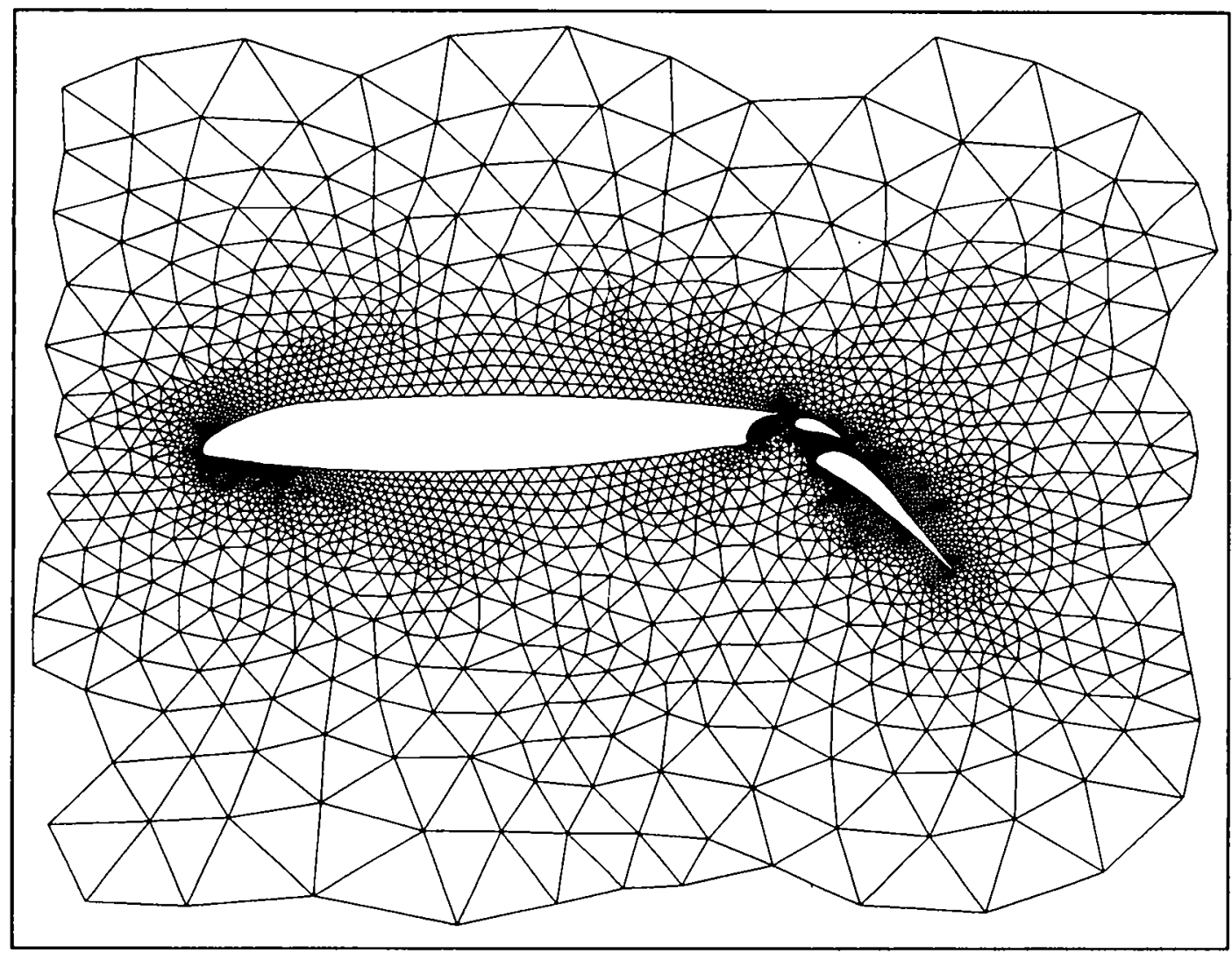

Fig. 1. Multi-element airfoil mesh.

Table 8 shows the results obtained by applying various partitioning methods to the dual of the multi-element airfoil graph. The methods are listed in rank order by hop-weight, which has been shown to closely correlate with the overhead due to 


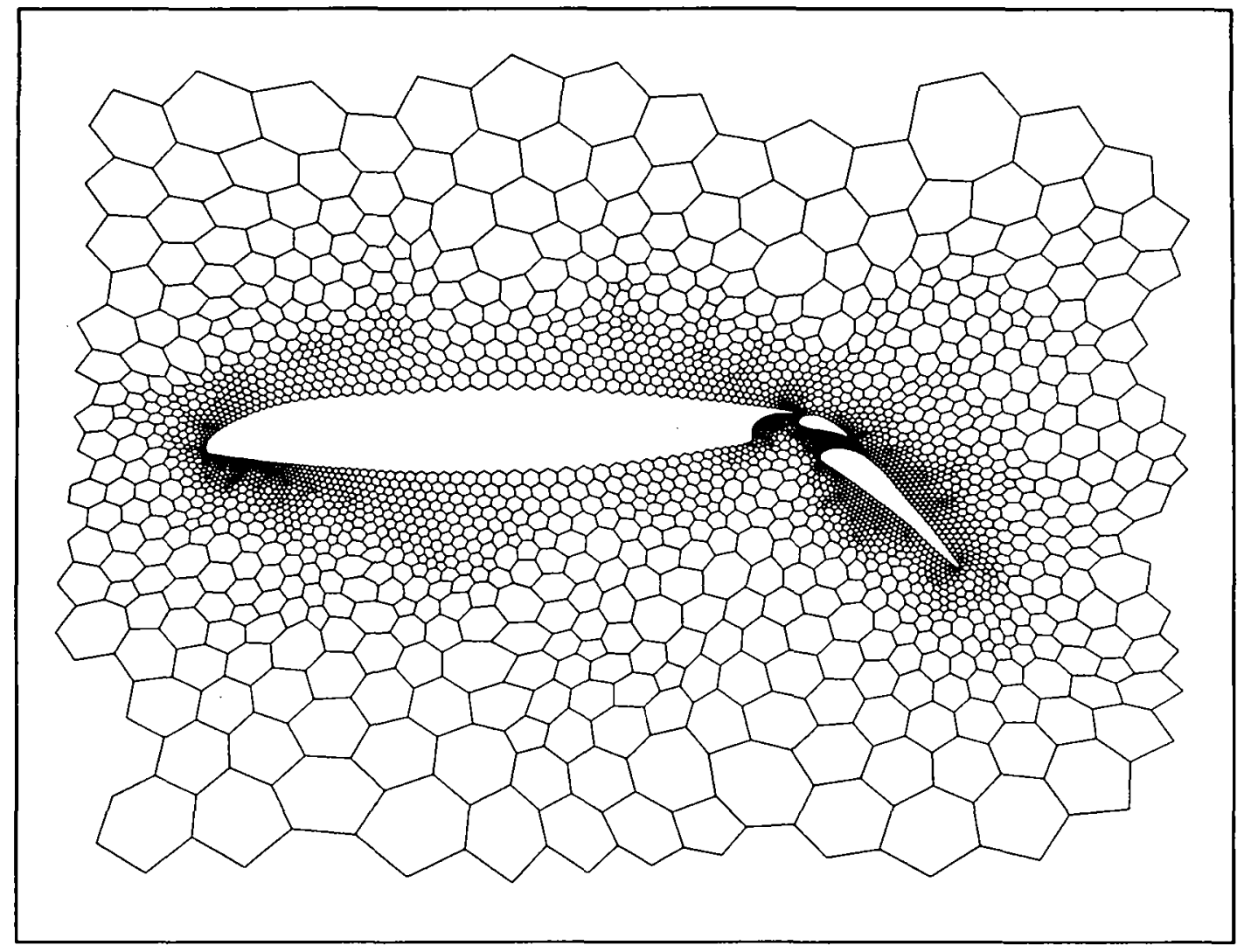

Fig. 2. Dual of multi-element airfoil mesh.

communication for the applications we are targeting [9]. A brief discussion of some important aspects of the partitioning algorithms follows.

$\mathrm{KL}$ refers to a recursive application of a version of the classic graph bisection heuristic devised by Kernighan and Lin [12]. KL must be supplied with an initial partition which is then improved by a greedy local strategy. We used an x-coordinate bisection of the vertices of the dual as an initial guess since this produced better partitions than any of the random initial guesses we tried. KL is a quick, linear time algorithm but is sensitive to the numbering of the vertices, and tends to do poorly on large problems because it only considers very local information about the graph. As with all bisection algorithms, one bit in the final processor assignment of a given vertex is determined at a time, so this algorithm makes no effort to minimize hops. It is clearly possible to add a phase to a bisection algorithm or any recursive partitioning algorithm which does try to further minimize hops by choosing an advantageous permutation of the set assignments of subgraphs. One such pivoting algorithm was detailed by Hammond [9]. We have used any pivoting strategy in our experiments.

The inertial method recently proposed by Nour-Omid [18] is also a recursive bisection method. It treats the mesh as a rigid structure and makes cuts orthogonal to the principle axis of the structure. This is also a fast algorithm which can be implemented to run in linear time, but requires geometric information which may be unavailable and, 


\begin{tabular}{||c||c|c||c|c||}
\hline \multirow{2}{*}{\multicolumn{1}{|c||}{ Method }} & \multicolumn{2}{c||}{8 Processors } & \multicolumn{2}{c||}{ 64 Processors } \\
\cline { 2 - 5 } & cuts & hops & cuts & hops \\
\hline \hline KL & 300 & 458 & 1158 & 2183 \\
\hline Inertial & 317 & 396 & 1166 & 1855 \\
\hline RSB & 212 & 286 & 997 & 1661 \\
\hline RSQ & & & 1030 & 1626 \\
\hline RSO & 221 & 224 & 1018 & 1463 \\
\hline RSOKL & 197 & 200 & 911 & 1287 \\
\hline
\end{tabular}

Table 1. Performance of different partitioning algorithms on dual of the multi-element airfoil mesh.

as the table indicates, it produces partitions of only moderate quality.

Recursive Spectral Bisection (RSB) is the name given by Simon to the $d=1$ spectral partitioning algorithm studied by him and others [18]. It requires no geometric information, is order insensitive and makes more sophisticated use of global information than the inertial method or KL. It produces much better partitions of large graphs than $\mathrm{KL}$ or inertial, but has an $O(n \sqrt{n})$ runtime dominated by the Lanczos iteration used to find the bisecting eigenvectors. Simon [18] and Williams [21] have both concluded that RSB is preferable to several partitioning strategies not considered here.

Recursive Spectral Quadrisection (RSQ) is our $d=2$ spectral partitioning algorithm. Here two bits in the final processor assignment are determined concurrently to approximately minimize hops in the corresponding two hypercube dimensions. This can be at the expense of a slight increase in the cut-weight, as the table indicates. Generally only a marginal number of additional Lanczos iterations are required to compute the second eigenvector, so RSQ is actually cheaper than two levels of RSB. In fact, if we assume that the cost of the eigenvector calculation is proportional to $n \sqrt{n}$ (which is appropriate for the Lanczos procedure [14]), then a single step of RSQ is faster than two steps of RSB by a factor of $1+\sqrt{2} / 2$. There is no 8 processor entry for RSQ because it is not possible to partition into 8 sets with an integral number of quadrisection steps.

Recursive Spectral Octasection (RSO) is our $d=3$ spectral partitioning algorithm which approximately minimizes hops in three hypercube dimensions at a time. In general it produces partitionings with fewer hops and perhaps slightly more cuts than RSQ and RSB, although it happens to do better on cuts than RSQ in this case. It is also cheaper than both RSQ and RSB. Assuming again that the eigenvector calculations cost $O(n \sqrt{n})$, one step of RSO is faster than three steps of RSB by a factor of $(3+\sqrt{2}) / 2$.

The last algorithm, RSOKL, is a composite algorithm in which the output of RSO at each stage of recursion is fed into a generalized KL algorithm capable of minimizing hops over an 8 way initial partitioning. The motivation for this strategy was to combine the global strength of RSO with the local finesse of KL. The resulting partition is clearly the best with respect to both cuts and hops. The KL phase of the algorithm accounts for only a small portion of the run time, so the net cost of RSOKL is substantially less than that of RSB. Notice that for the 8 processor case the cut and hop totals are nearly equal, indicating that almost all communication occurs between adjacent processors. 
KL can be appended to the other algorithms as well, but we have found RSOKL to be the best combination given our communication metric. RSOKL is discussed in more detail in [10].

RSO and RSQ can also be more robust than RSB when the graph exhibits symmetry. For example, the three-fold symmetry of the cubic grid-graph causes $\lambda_{2}$ of its Laplacian to have a multiplicity of three, and the corresponding eigenspace to be three-dimensional. Since RSB chooses a single vector from this subspace essentially at random, it may fail badly. It will, for example, make a diagonal cut through the grid for some Lanczos starting vectors. In contrast, RSO works within the entire subspace, rotating the basis vectors in such a way that it returns a gray-coding of blocks of the grid. This is the optimal result in which cuts and hops are as small as possible and all communication is between adjacent processors.

To demonstrate that this discrepancy does arise in practice, we ran both methods on a simple $4 \times 4 \times 4$ grid graph. In RSB we used the Lanczos starting vector recommended by Pothen, Simon and Liou [15], namely $r_{i}=i-(n+1) / 2$, and iterated until the eigenresidual $A u-\lambda u$ was below $10^{-6}$. The resulting decomposition had 72 cuts and 78 hops. In RSO we solved to the same accuracy for this starting vector and several random starting vectors. In each case we obtained 48 cuts and 48 hops, the optimal partitioning. Similar results may be observed with other symmetric graphs.

9. Conclusions. We have presented a method for mapping large problems onto the nodes of a hypercube multiprocessor in such a way that the computational load is balanced and the communication overhead is kept small. For the problems we have investigated, this approach generates mappings that have lower communication requirements than other partitioning techniques. Because the 2nd and 3rd eigenvectors are relatively inexpensive to calculate, the net cost of spectral quadrisection or octasection is significantly less than that of spectral bisection. In addition, our method yields computable lower bounds for the communication cost of any balanced partitioning scheme which are tighter than those previously known.

Although our method was developed with a hypercube communication network in mind, this approach should work well for other machine topologies. For example, a two-dimensional mesh can be defined as a collection of two-dimensional hypercubes, so a recursive application of our quadrisection approach is immediately applicable. Similarly, a three-dimensional mesh is composed of three-dimensional hypercubes, so our octasection algorithm can be applied. For other architectures we expect our approach to be useful as a heuristic. Although the method tries to minimize a communication function that counts hypercube hops, in practice the spectral quadrisection and octasection algorithms divide a domain into pieces that require a small communication volume. This should lead to low communication overhead on most parallel machines.

Graph partitioning also finds application in network design, circuit layout, sparse matrix computations and a number of other disciplines. Consequently, the partitioning algorithm we have described may find uses far afield from parallel computing. More broadly, the way we have made use of multiple eigenvectors is, to our knowledge, unlike any previous work in spectral graph theory. It is our hope that these ideas can be 
applied to other spectral graph theoretic problems.

Acknowledgements. The ideas in this paper have evolved through discussions with many people including Horst Simon, Alex Pothen, Louis Romero, Ray Tuminaro, John Shadid and Steve Plimpton.

\section{REFERENCES}

[1] T. Barth. Personal Communication, December 1991.

[2] R. Bopana, Eigenvalues and graph bisection: An average case analysis, in Proc. 28th Annual Symposium on Foundations of Computer Science, IEEE, 1987, pp. 280-285.

[3] W. Donath and A. Hoffman, Algorithms for partitioning of graphs and computer logic based on eigenvectors of connection matrices, IBM Technical Disclosure Bulletin, 15 (1972), pp. 938944.

[4] - Lower bounds for the partitioning of graphs, IBM J. Res. Develop., 17 (1973), pp. 420-425.

[5] M. FIEDlER, Algebraic connectivity of graphs, Czechoslovak Math. J., 23 (1973), pp. 298-305.

[6] - A property of eigenvectors of nonnegative symmetric matrices and its application to graph theory, Czechoslovak Math. J., 25 (1975), pp. 619-633.

[7] R. Fletcher, Practical Methods of Optimization, Volume 2, Constrained Optimization, John Wiley \& Sons, New York, 1986.

[8] M. GaReY, D. Johnson, AND L. STOCKMEYer, Some simplified NP-complete graph problems, Theoretical Computer Science, 1 (1976), pp. 237-267.

[9] S. HAMMOND, Mapping unstructured grid computations to massively parallel computers, PhD thesis, Rensselear Polytechnic Institute, Dept. of Computer Science, Renssealear, NY, 1992.

[10] B. Hendrickson and R. Leland, Domain mapping of parallel scientific computations, Tech. Rep. SAND 92-1461, Sandia National Laboratories, Albuquerque, NM, 1992.

[11] J. J. Dennis AND R. SChNaBEL, Numerical methods for unconstrained optimization and nonlinear equations, Prentice-Hall, Inc., Englewood Cliffs, NJ, 1983.

[12] B. KERNIGHAN AND S. LIN, An efficient heuristic procedure for partitioning graphs, Bell System Technical Journal, 29 (1970), pp. 291-307.

[13] В. МонаR, The Laplacian spectrum of graphs, in 6th International Conference on Theory and Applications of Graphs, Kalamazoo, MI, 1988.

[14] B. Parlett, The Symmetric Eigenvalue Problem, Prentice-Hall, Englewood Cliffs, NJ, 1980.

[15] A. Pothen, H. Simon, AND K. Liou, Partitioning sparse matrices with eigenvectors of graphs, SIAM J. Matrix Anal., 11 (1990), pp. 430-452.

[16] D. POWERs, Graph partitioning by eigenvectors, Lin. Alg. Appl., 101 (1988), pp. 121-133.

[17] F. RENDL AND H. Wolkowicz, A projection technique for partitioning the nodes of a graph, Tech. Rep. CORR 90-20, University of Waterloo, Faculty of Mathematics, Waterloo, Ontario, November 1990.

[18] H. Simon, Partitioning of unstructured problems for parallel processing, in Proc. Conference on Parallel Methods on Large Scale Structural Analysis and Physics Applications, Pergammon Press, 1991.

[19] P. SUARIS AND G. KEDEM, An algorithm for quadrisection and its application to standard cell placement, IEEE Trans. Circuits and Systems, 35 (1988), pp. 294-303.

[20] T. TokUYama and J. NaKano, Geometric algorithms for a minimum cost assignment problem, in Proc. 7th Annual Symposium on Computational Geometry, ACM, 1991, pp. 262-271.

[21] R. Williams, Performance of dynamic load balancing algorithms for unstructured mesh calculations, Concurrency, 3 (1991), pp. 457-481.

[22] D. Womble, A time-stepping algorithm for parallel computers, SIAM J. Sci. Stat. Comput., 11 (1990), pp. 824-837. 
DISTRIBUTION :

Scott Baden

University of California, San Diego

Dept. of Computer Science

9500 Gilman Drive

Engineering 0114

La Jolla, CA 92091-0014

Steve Barnard

NAS Systems Division

Applied Research Branch

NASA Ames Research Center

Mail Stop T045-1

Moffett Field, CA 94035

Edward Barragy

Dept. ASE/EM

University of Texas

Austine, TX 78712

M. Bexzins

Scool of Computer Studies

The University of Leeds

Leeds, LS290T

United Kingdom

Rob Bisseling

Shell Research B.V.

Postbus 3003

1003 AA Amsterdam

The Netherlands

Ravi Boppana

Department of Computer Science

NYU

251 Mercer Street

New York, NY 10012

$J$. Browne

University of Texas

Dept. of Computer Science

Taylor Hall 5.126

Austin, TX 78712

Tony Chan

Department of Computer Science

The Chinese University of Hong Kong

Shatin, NT

Hong Kong

Ted Charrette

MIT BIdg. E3 554

42 Carleton St.

Cambridge, MA 02142

Siddartha Chatter jee

RIACS, NASA Ames Research Center

Mail Stop T045-1

Moffett Field, CA 94035-1000

Tom Coleman

Dept. of Computer Science

Upson Hall

Cornell University

Ithaca, NY 14853 
919 E. Hillsdale Blvd.

Foster City, CA 94404

Alan Edelman

University of California, Berkeley

Dept. of Mathematics

Berkeley, CA 94720

Salvatore Filippone

IBM ECSEC

Viale Oceano Pacifico 171/173

00144 Roma, Italy

John Gilbert

Xerox PARC

3333 Coyote Hill Road

Palo Alto, CA 94304

Bashkar Ghosh

Department of Computer Science

Yale University

POB 2158, Yale Station

New Haven, CT 06520

Anne Greenbaum

New York University

Courant Institute

251 Mercer Street

New York, NY 10012-1185

Steve Hammond

CERFACS

42 Ave Gustave Coriolis

31057 Toulouse Cedex

France

Mike Heath

University of Illinois

4157 Beckman Institute

$405 \mathrm{~N}$. Mathews Ave.

Urbana, IL 61801

Greg Heileman

EECE Department

University of New Mexico

Albuquerque, NM 87131

A.J. Hey

University of Southampton

Dept. of Electronics and Computer Science

Mountbatten Bldg., Highfield

Southampton, SO9 5NH

United Kingdom

Adolfy Hoisie

Cornell University

Cornell Theory Center

631 E\&TC Bldg

Ithaca, NY 14853

Graham Horton

Universitat Erlangen-Nurnberg

IMMD III

Martensstrase 3

8520 Erlangen, FRG 
Kapil Mathur

Thinking Machines Corporation

245 First Street

Cambridge, MA 02142-1214

William McColl

Oxford University Computing Laboratory

8-11 Keble Road

Oxford, OXI 3QD

United Kingdom

Robert McLay

University of Texas at Austin

Dept. ASE-EM

Austin, TX 78712

Jill Mesirov

Thinking Machines Corporation

245 First street

Cambridge, MA 02142-1214

Bojan Mohar

Department of Mathematics

University of Ljubljana

Jadranska 19, 61111 L jublajana

Slovenia

Can Ozturan

Deptartment of Computer Science

Rensselaer Polytechnic Institute

Troy, NY 12180

Glauscio Paulino

Civil Engineering

Cornell University

Hollister Hall 413

Ithaca, NY 14853

Paul Plassman

Math and Computer Science Division

Argonne National Lab

Argonne, IL 60439

Alex Pothen

Computer Science Department

University of waterloo

waterloo, Ontario N2L 3G1

Canada

Mike Quayle

Cadence Design Systems

2 Lowell Research Center Drive

Lowell, MA 01857

San jay Ranka

School of Computer and Information Science

Suite 4-116

Center for Science and Technology

Syracuse, NY 13244-4100

Satish Rao

NEC Research Institute,

4 Independence Way,

Princeton, NJ, 08540 


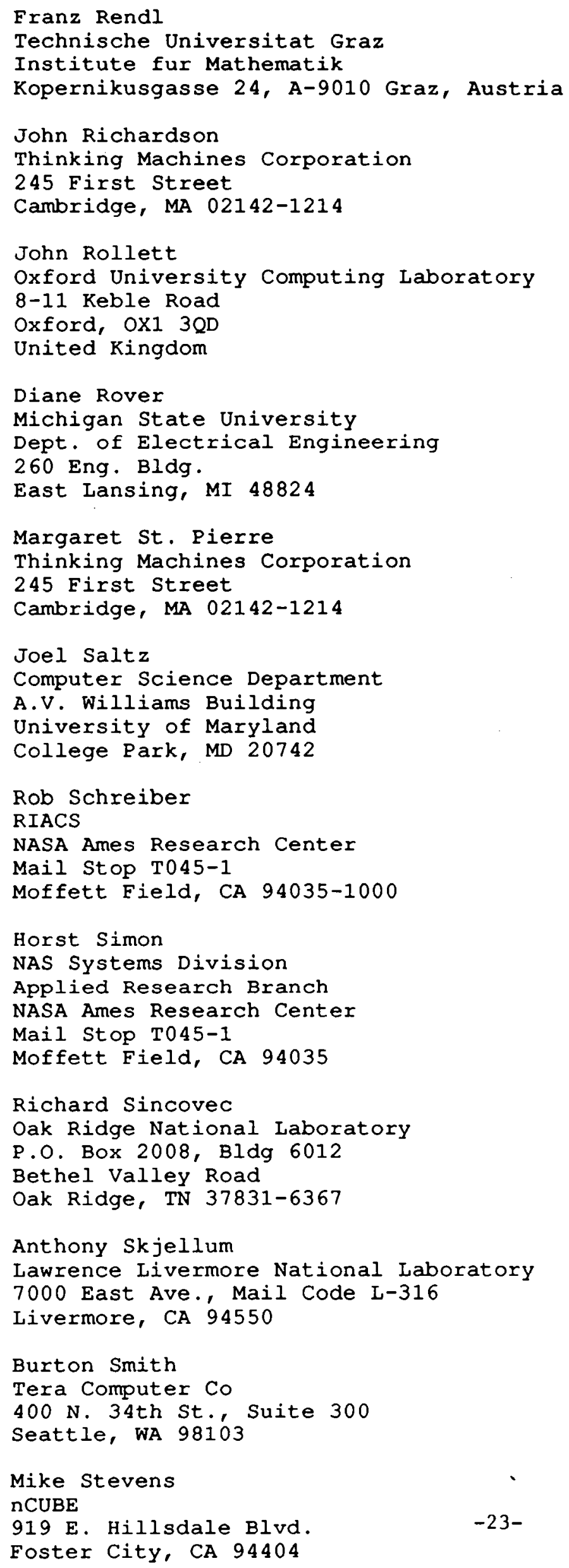


Judy Sturtevant

Mission Research Corporation

1720 Randolph Rd. SE

Albuquerque, NM 87106-4245

Shari Trewin

Edinburgh Parallel Computing Centre

The University of Edinburgh

Janes Clerk Maxwell Bldg.

The King's Buildings

Mayfield Road

Edinburgh, EH $93 \mathrm{Jz}$

United Kingdom

Ray Tuminaro

CERFACS

42 Ave Gustave Coriolis

31057 Toulouse Cedex

France

Stefan Van de Walle

Katholieke Universiteit Leuven

Dept. of Computer Science

Celest jnenlaan 200A

B-3001 Leuven, Belgium

John Van Rosendale

ICASE, NASA Langley Research Center

MS $132 \mathrm{C}$

Hampton, VA 23665

Steve Vavasis

Dept. of Computer Science

Upson Hall

Cornell University

Ithaca, NY 14853

C. Walshaw

School of Computer Studies

University of Leeds

Leeds LS2 9JT

United Kingdom

Robert Weaver

University of Colorado at Boulder

Dept. of Computer Science

Campus Box 430

Boulder, CO 80309

Roy Williams

California Institute of Technology

206-49

Pasadena, CA 91104

Henry Wolkowicz

Department of Combinatorics and Optimization

University of Waterloo

Waterloo, Ontario, N2I $3 G 1$

Canada 


$\begin{array}{ll}\text { Sudip Dosanjh } & 1402 \\ \text { Bill Camp } & 1421 \\ \text { Doug Cline } & 1421 \\ \text { David Gardner } & 1421 \\ \text { Grant Heffelfinger } & 1421 \\ \text { Scott Hutchinson } & 1421 \\ \text { Martin Lewitt } & 1421 \\ \text { Steve Plimpton } & 1421 \\ \text { Mark Sears } & 1421 \\ \text { John Shadid } & 1421 \\ \text { Julie Swisshelm } & 1421 \\ \text { Dick Allen } & 1422 \\ \text { Bruce Hendrickson (15) } & 1422 \\ \text { David Womble } & 1422 \\ \text { Ernie Brickell } & 1423 \\ \text { Robert Benner } & 1424 \\ \text { Carl Diegert } & 1424 \\ \text { Art Hale } & 1424 \\ \text { Rob Leland (15) } & 1424 \\ \text { Courtenay Vaughan } & 1424 \\ \text { Steve Attaway } & 1425 \\ \text { Johnny Biffle } & 1425 \\ \text { Mark Blanford } & 1425 \\ \text { Jim Schutt } & 1425 \\ \text { Michael McGlaun } & 1431 \\ \text { Allen Robinson } & 1431 \\ \text { Paul Yarrington } & 1432 \\ \text { David Martinez } & 1434 \\ \text { Dona Crawford } & 1900 \\ \text { William Mason } & 1952 \\ \text { Technical Library (5) } & 7141 \\ \text { Technical Publications } & 7151 \\ \text { Document Processing for } & \\ \text { DoE/OsTI (10) } & 7613-2 \\ \text { Central Technical File } & 8523-2 \\ \text { Charles Tong } & 8117 \\ & \end{array}$

